\title{
Pass-thoughts Authentication System based on EEG Signals Using Artificial Neural Network
}

\author{
Amer A. Sallam
}

Department of Computer Networks \& Distributed Systems, Faculty of Engineering \& IT, Taiz University, Taiz, Yemen amer.sallam@taiz.edu.ye

Amin Saif

Department of Mechatronics Engineering \& Robotics, Faculty of Engineering \& IT, Taiz University, Taiz, Yemen amye008@gmail.com

Mogeeb A. Saeed

Department of Computer Networks \& Distributed Systems, Faculty of Engineering \& IT, Taiz University, Taiz, Yemen mogeeb1982@gmail.com

Siham A. Mohammed

Department of Information Technology, Faculty of Engineering \& IT, Taiz University, Taiz, Yemen siham.alkadasi@gmail.com

-ABSTRACT-

Authentication with textual password has several limitations: passwords have low entropy in practice, are often difficult to remember, are vulnerable "shoulder surfing". Biometric system does not meet requirement as well. It relies upon unchanging features that have a lifetime as long as the individual. To avoid this limitation, we start to authenticate with thinking pass thought. User performs one mental task such as thinking of a word or phrase. In this study, Electroencephalography (EEG) was used as method for monitoring and recording the electrical activity of the brain. These signals can be captured and processed to get the useful information that can be used in pas-thoughts authentication system. Suitable analysis is essential for EEG to differentiate between best and worst tasks used for authentication. This study focuses on usefulness of EEG signal to identify best tasks suitable for the pass-thoughts authentication system. Artificial neural network (ANN) is used to train the data set. Then tests are conducted on the testing data of EEG signal to identify best and worst tasks suitable for authentication. Finally, the system performance was evaluated by computing the accuracy and therefore promising results were obtained.

Keywords - Electroencephalography; Artificial Neural Network; Discrete Wavelet Transform.

Date of Submission: July 23, 2019

Date of Acceptance: Aug 14, 2019

\section{INTRODUCTION}

The Brain-Computer-Interface (BCI) is a communication system that enables human to interact with their surrounding without involving of nerves and muscles by using control signals generated from electroencephalographic activity (EEG).

EEG is typically noninvasive, with the electrodes placed along the scalp, although invasive electrodes are sometimes used in specific applications [1][2][3]. EEG measures voltage fluctuations resulting from ionic current within the neurons of the brain. EEG signals can be composed into five EEG sub-bands (Delta, theta, Alpha, Beta, and gamma) [4][5]. As illustrated in Fig. 1.

Delta waves are slow, loud brainwaves. They are generated in deepest meditation and dreamless sleep. emitting between $0.1 \mathrm{~Hz}$ and $4 \mathrm{~Hz}$ cycles per second. Alpha brainwaves are dominant during quietly flowing thoughts, and in some meditative states, its frequencies are around $7 \mathrm{~Hz}$ to $13 \mathrm{~Hz}$ pulses per second.

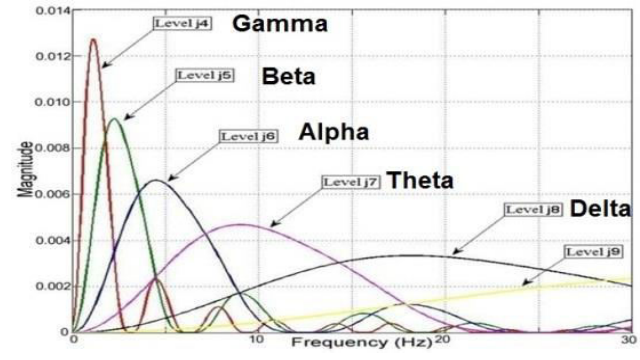

Fig. 1. Brainwaves frequencies

Beta brainwaves dominate our normal waking state of consciousness when attention is directed towards cognitive tasks and the outside world, with frequencies ranging from $13 \mathrm{~Hz}$ to $60 \mathrm{~Hz}$ pulses per second. Gamma brainwaves are the fastest of brain waves its frequency range is from 30 $\mathrm{Hz}$ to $60 \mathrm{~Hz}$ [6].

\section{LITERATURE REVIEW}

Several works have been done on pass-thoughts authentication using different techniques. Moran et al. [7] developed a model to examine the system for robustness against deliberate attacks thought impersonators, Johnson et al. [8] this study is attuned toward the extent to which system can be broken. If an attacker knows your pass- 
thought, and puts on the brain reader, what is now the likelihood that they can successfully authenticate as you? Their overall goal was to investigate the susceptibility of brainwave signal authentication to impersonation attacks; and a key technical objective was to measure the extent to which the attacker is aided by more information about the defender's pass-thoughts processed in an accurate way.

Chuang et al. [9] this study has focused on usability and performance of user authentication using consumergrade EEG sensor technology. By choosing custom tasks and custom acceptance thresholds for each subject; they can achieve 99\% authentication accuracy using singlechannel EEG signals. They also explored the usability of different classes of mental tasks.

Baby et al. [10] discussed the ability to effectively classify electroencephalograms (EEG) is the foundation for building usable Brain-Computer Interfaces. In their work EEG signals were used to extract the information and classify the different mental task. This data contains recording of 5 subjects in different mental task conditions. For classification of these mental tasks wavelet was used to extract the features. Kumar et al. [11] this study has showed the case for authentication using brainwaves is made yet stronger by the availability of consumer-grade brainwave sensors and their integration with wearable devices (e.g., integration with augmented reality glasses, as these typically do not have keyboards, and often times do not even have a touchscreen, making password-based and PIN-based authentication difficult.

Curran et al. [12] proposed a model for Pass Thoughts Authentication with Low Cost Ear-EEG. This study tried to explore how wearable computing devices could be mirrored in an authentication scheme based on electroencephalography (EEG) signals which collected from the ear. they have evaluated their model using a low cost, single-channel, consumer grade device for data collection. they achieve a $44 \%$ reduction in half total error rate (HTER) compared with a random classifier, corresponding to a $72 \%$ authentication accuracy in withinparticipants analyses and a $60 \%$ reduction and $80 \%$ accuracy in between-participant analyses.

Hosni et al. [13] used classification of EEG signals using different feature extraction techniques for mental-task BCI, this study proposed a model that try to help paralyzed people communicating with others by using their electroencephalogram (EEG) signals. during this study, EEG signals from 5 mental tasks were recorded from 7 subjects and combinations of 2 different mental tasks were studied for each subject for one trial. The motivation of this work was using linear predictive Coding (LPC) method to compress channels of EEG into one channel. Eight features are employed for each signal of EEG using LPC 1st order followed by 3 levels of Discrete Wavelet Transform (DWT). New mother wavelet is used to be near the waveform of EEG signals. Statistical calculations are conducted for the 4 coefficients of DWT. Classification is conducted using support vector machine SVM. The classifier using SVM provided a high recognition rate reaching up to $100 \%$, in some cases, and an average rate of about $85 \%$. The average specificity percent is $83.33 \%$. The average sensitivity percent is $86.66 \%$.

In the proposed work, the dataset is divided into two groups - training and testing sets. First, EEG signals are decomposed into five sub-band signals using Discrete Wavelet Transformation (DWT) that widely used in frequency-domain transformation [13] [14]. Then, ANN used to train the data. Finally, the tests are conducted on the testing sets to identify the given EEG signal as best or worst mental tasks. We used EEG signals obtained from BCI Competition III - Berlin Brain-Computer Interface dataset [16] to conduct the experiments. The rest of the paper is organized as follows. Section 2 provides the methodology of the work. Section 3 presents the test results and finally, section 4 concludes the paper.

\section{METHODOLOGY}

To address the authentication limitations and vulnerabilities that existed in both textual password and biometric system, an EEG signal classification with ANN is proposed and illustrated by Figs. 2-5. Fig. 1 shows how the signal segments are trained with the known output or target values, in which ANN neuron weights are computed.

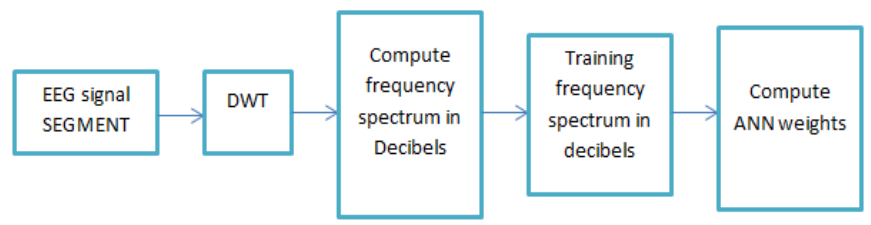

Fig. 2. Training process of EEG signal segments

During the training process the model investigates and compares the performance of the brain signals and their extracted features through the following steps:

- Data Acquisition: This step concern on capturing and preparing the dataset of the brain signals that obtained from BCI Competition III - Berlin Brain-Computer Interface dataset [16]

- Data Pre-processing and Feature Extraction: Pre-process the power spectrum data to compress the samples in the temporal dimension. Thus, signal segments go through DWT filter bank process to transform into the five frequency ranges. Then the frequency spectrum in Decibel at different frequency ranges are calculated and averaged. and noise elimination processes will be applied for each recorded signal. Then, DWT used to determine those components of the signal that are deemed most relevant and important by examining the raw brain signals (i.e.; Alpha and Beta) and their extracted features values ( $\max$, min, mean). The Discrete Wavelet Transform (DWT) algorithm is used for this purpose. So, the generated password task that is represented by the raw signal of EEG training dataset has been decompose into five frequency bands (Alpha, 
Beta, Delta and Theta and gamma) through DWT as illustrated In Fig. 3, and Table 1.

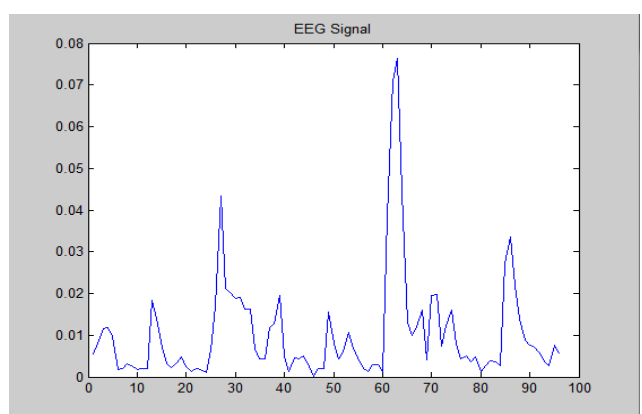

Fig. 3. DWT is applied to a training dataset

Table 1. The decomposition of EEG signal

\begin{tabular}{|c|c|c|c|}
\hline 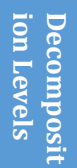 & 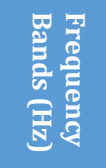 & 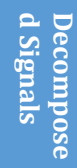 & 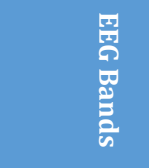 \\
\hline $\begin{array}{l}1 \\
2 \\
3 \\
4 \\
5 \\
5\end{array}$ & $\begin{array}{c}64-128 \\
32-64 \\
16-32 \\
8-16 \\
4-8 \\
0-4\end{array}$ & $\begin{array}{l}\text { D1 } \\
\text { D2 } \\
\text { D3 } \\
\text { D4 } \\
\text { D5 } \\
\text { A5 }\end{array}$ & $\begin{array}{c}\text { Higher } \\
\text { gamma and } \\
\text { noise } \\
\text { Lower } \\
\text { gamma }(\gamma) \\
\text { Beta }(\beta) \\
\text { Alpha }(\alpha) \\
\text { Theta }(\theta) \\
\text { Delta }(\delta)\end{array}$ \\
\hline
\end{tabular}

\section{- Classification:}

last step takes care of analyzing the features in order to determine what specific action should be taken based on the given values of the features. The backpropagation neural network algorithm was chosen as a classifier to find out the best accuracy. Fig. 4 presents the ANN diagram performed in Matlab. Fifteen neurons are used in the hidden layer; feedforward back-propagation algorithm was used where the number of output was kept as five possible outputs for each task (i.e.; $T 7$ as $[7,7,7,7,7], T 3$ as $[3,3,3,3,3]$ and $T 2$ as $[2,2,2,2,2])$.

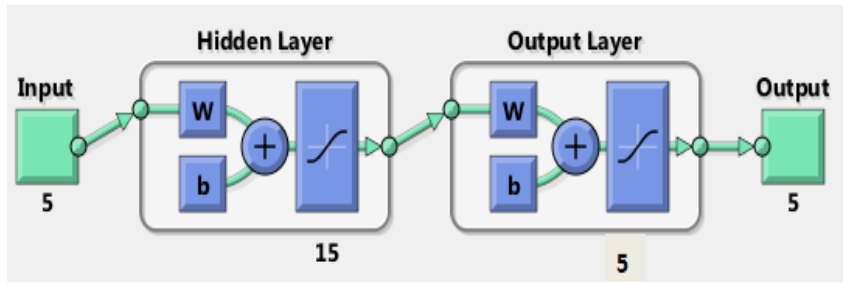

Fig. 4. ANN diagram for EEG signal segments

In Fig. 5, testing procedure of EEG signal segments is presented. Similar to training process, signals first undergo DWT filter bank process to split different frequency ranges, followed by computation of frequency spectrum which is then averaged over each range. This results in five values of frequency spectrum in Decibel, providing the inputs of ANN. Next, the results are computed using the same weights as obtained in training stage. Finally, the output is classified as mental tasks class.

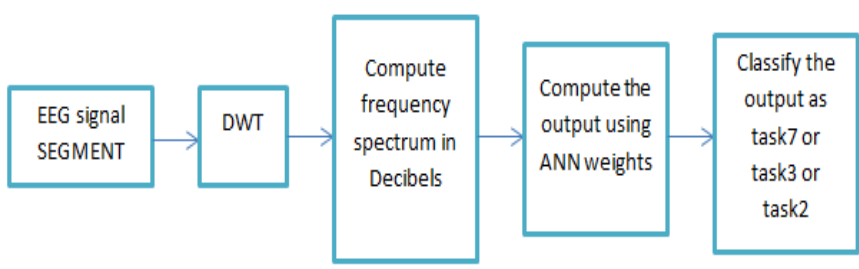

Fig.5. Testing process of EEG signal segments

During the training phase, the neural weights in ANN are calculated and they are used in testing phase. The output from the testing phase is obtained as $\mathrm{T}=[\mathrm{T} 1, \mathrm{~T} 2, \mathrm{~T} 3, \mathrm{~T} 4$,$] .$ Using the output, we find the decision $\mathrm{D}$ by:

$$
D=\left\{\begin{array}{c}
0, \text { if authenticated } \\
1, \text { if rejected }
\end{array}\right.
$$

The decision parameter determines whether the EEG signal segment is authenticated or rejected. Specifically, if $\mathrm{D}=0$, the signal segment is authenticated, and if $\mathrm{D}=1$ indicates the segment is rejected. After testing with different sample sets, the accuracy of the proposed method is computed as:

$$
\mathrm{HTER}=(\mathrm{FAR}+\mathrm{FRR}) / 2
$$

where FAR is False Acceptance Rate which occur when the system accepts an impostor, $F R R$, False Rejection Rate which occur when the system rejects an authorized user. HTER Half Total Error Rate which form the performance of an authentication system.

\section{RESUlTS AND DISCUSSIONS}

In the previous sections we explore and discuss how the brain signals could be mirrored in an authentication scheme using different mental task. The goal of a passthought system is to extract as much entropy as possible from a user's brain signals upon transmitting a thought. In the proposed work the data set used contains data of three mental tasks as illustrated in Table 2 .

TABLE 2. List of the mental tasks.

\begin{tabular}{|lll|}
\hline Task & Description & $\begin{array}{l}\text { Class } \\
\text { Name }\end{array}$ \\
\hline T7 & $\begin{array}{l}\text { Generation of words } \\
\text { beginning with the same } \\
\text { random letter }\end{array}$ & 7 \\
\hline T3 & $\begin{array}{l}\text { Imagination of repetitive self- } \\
\text { paced right-hand movements }\end{array}$ \\
\hline T2 & $\begin{array}{l}\text { Imagination of repetitive self- } \\
\text { paced left-hand movements }\end{array}$ \\
\hline
\end{tabular}

Our aim was to compare between the three tasks and show which one is the best for the authentication system. Analysis of the brain signals using Electroencephalography (EEG) is used for identifying best 
and worst tasks suitable for pass-thoughts authentication system.

In the tests, we use an EEG dataset from (BCI Competition III - Berlin Brain-Computer Interface dataset) to verify our method. The dataset contains 12 signals with different mental tasks. We trained the dataset with two or more tasks per signal and tested on 320 hours of continuous scalp EEG sampled at $256 \mathrm{~Hz}$. The EEG samples are split into sets of 4 sessions of a given subject were acquired on the same day, each lasting 4 minutes with 5-10 minutes breaks in between them. The subject performed a given task for about 15 seconds and then switched randomly to another task at the operator's request. We conducted three experiments namely, E1, E2 and $E 3$ and the experimental setup and the overall results are shown in Table 3. In the columns two and three, the training and testing sets are for different mental tasks cases are given. Finally, the accuracy obtained from each experiment is presented.

TABLE 3. Accuracy of the test results.

\begin{tabular}{|c|c|c|c|c|c|c|c|c|}
\hline \multirow{2}{*}{$\begin{array}{c}\text { Experi } \\
\text { ment }\end{array}$} & \multicolumn{3}{|c|}{ Number of training sets } & \multicolumn{3}{|c|}{ Number of testing sets } & \multicolumn{2}{|c|}{ Accuracy } \\
\hline & T3 & T2 & T7 & T3 & T2 & T7 & $\begin{array}{l}\text { Best } \\
\text { Task }\end{array}$ & $\begin{array}{c}\text { Worst } \\
\text { Task }\end{array}$ \\
\hline E1 & 150 & 150 & 150 & 150 & 150 & 150 & $96 \%$ & $60 \%$ \\
\hline E2 & 300 & 300 & 300 & 300 & 300 & 300 & $98 \%$ & $65 \%$ \\
\hline E3 & 1500 & 1500 & 1500 & 1500 & 1500 & 1500 & $100 \%$ & $70 \%$ \\
\hline
\end{tabular}

The detailed results are given in table 4. First column provides the task name; Second column provides the sample name where the first two character imply the experiment number and the last two characters imply the sample identification number. For example, E1S3 implies the sample is from experiment El with the sample identification number S3. Columns three to six present the output values of $T$. The last column provides the class of each task as given in the dataset (i.e.; 7 indicates $T 7,3$ indicates $T 3$ and 2 indicates $T 2$ ). As we can see $T 2$ has obtained the best accuracy comparing to the other tasks.

TABLE 4. Decision from experimental results and the actual results from some samples

\begin{tabular}{|c|c|c|c|c|c|}
\hline Task & sample & T1 & T2 & T3 & $\mathrm{T} 4$ \\
\hline \multirow{3}{*}{ T7 } & E1S1 & 7 & 7 & 7 & 7 \\
\hline & E1S2 & 6.9 & 7 & 5 & 6.9 \\
\hline & E1S3 & 6.9 & 7 & 7 & 6.9 \\
\hline \multirow{3}{*}{ T3 } & E2S1 & 2 & 3 & 2 & 3 \\
\hline & E2S2 & 3 & 3 & 5 & 3 \\
\hline & E2S3 & 3 & 3 & 3 & 3 \\
\hline \multirow{3}{*}{ T2 } & E3S1 & 2 & 2.0003 & 2 & 2 \\
\hline & E3S2 & 2 & 2 & 2 & 2 \\
\hline & E3S3 & 2 & 2 & 2 & 2 \\
\hline & $\begin{array}{l}\text { Highest } \\
\text { Highest } \\
\text { Highest }\end{array}$ & $\begin{array}{l}\text { for } 7 \\
\text { for } 7 \\
\text { for T }\end{array}$ & & & $\begin{array}{l}\text { value } \\
\text { value } \\
\text { value }\end{array}$ \\
\hline
\end{tabular}

In experiment $E 1$, where the network was trained with 150 cases for all mental tasks, the result is illustrated in Fig. 6. The performance which represents the deviation between network output and the target is shown in Fig. 6. While the Regression $(R)$ represents the percentage of similarity between network output and network target. Best signal was beta which is represented by $T 2$ and best feature was max mean which was applied with E1S1.
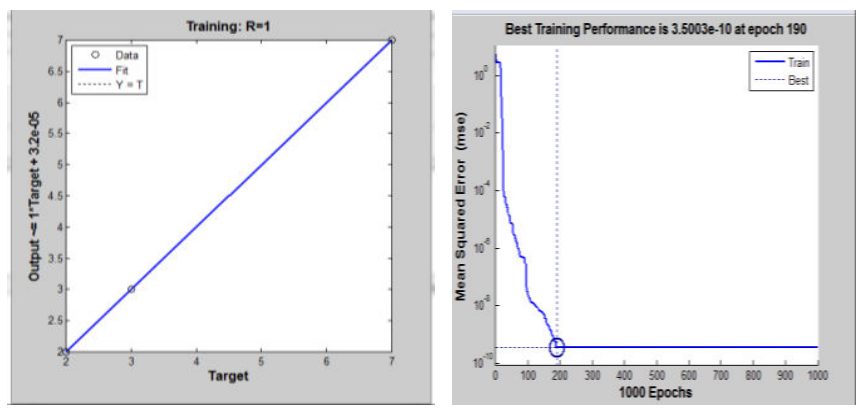

Fig. 6. Training performance and regression results for experiment E1

Experiment E2, which consider 300 cases for cases for all mental tasks, it shows a better accuracy; however, the network performance is lower with more regression value as shown in Fig. 7. Best signal was beta and theta which is represented by $T 2$ and $T 3$ while best feature was mean which was applied with E2S3.

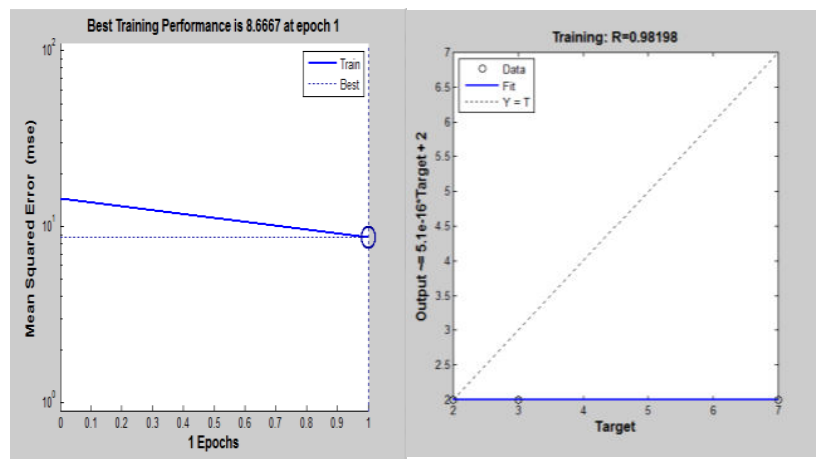

Fig. 7. Training performance and regression results for experiment E2.

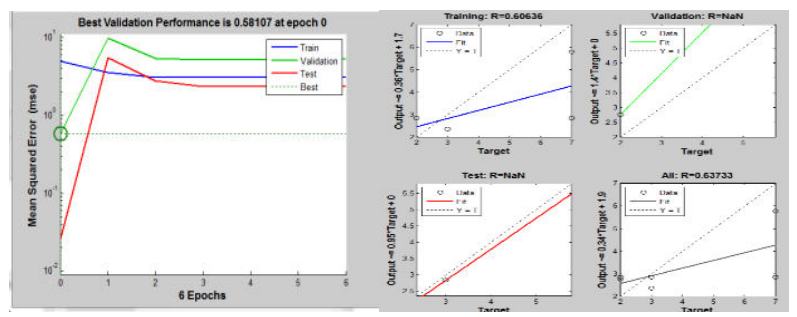

Fig. 8. Training performance and regression results for experiment E3

Results in experiment $E 3$ with 1500 cases for cases for all mental tasks, $T 2$ mental task provides the heights accuracy but worst in terms of performance and regression as presented in Fig. 8. Best signal was theta which is represented by $T 3$ and best feature was mean which was applied with E3S3. So, we find out that theta and mean were best signal and feature suitable for the authentication. The authentication problem is also referred to as the user verification problem. Given an (identity, sample) pair, the authentication system must determine if the sample provides a legitimate match to the identity. Authentication systems make two types of errors: False Acceptance (FA) errors occur when the system accepts an impostor, while False Rejection (FR) errors occur when the system rejects an authorized user. The performance of an authentication system can thus be measured in terms of its False 
Acceptance Rate (FAR) and False Rejection Rate (FRR). The two error measures are often merged to form the Half Total Error Rate (HTER), defined as:

$$
\mathrm{HTER}=(\mathrm{FAR}+\mathrm{FRR}) / 2
$$

for each participant for each task, 12 samples were randomly selected as training set $\mathrm{A}$, and 12 samples as testing set A. Also 12 were randomly selected as training set $\mathrm{B}$, and another 12 as testing set $\mathrm{B}$. The ANN was then trained on classifying between training set A as a model of correct authentication, and training set $\mathrm{B}$ as a model of correct rejection. The FRR was calculated by testing the $A N N$ on testing set A (samples from the same task), resulting in a list of 12 0's (authenticate) and 1's (reject) and taking the mean of this list. Similarly, the FAR was calculated by testing the trained $\mathrm{ANN}$ on testing set B (samples from other tasks) and taking 1 minus the mean of the resulting list of 0's and 1's. Finally, the HTER was calculated by averaging the $F R R$ and $F A R$ for each task/participant pair. All is illustrated in table 5 .

TABLE 5. Authentication results, means across participants with standard errors.

\begin{tabular}{|llll|}
\hline Task & FAR & FRR & HTER \\
\hline T7 & 0.83 & 0.08 & 0.45 \\
\hline T3 & 0.83 & 0.25 & 0.54 \\
\hline T2 & 0.5 & 0.00 & 0.25 \\
\hline
\end{tabular}

As shown in table 4 the $T 2$ task performed best with an HTER of 0.25 followed by the $T 7$ task tied at 0.45 .

\section{CONCLUSION AND FUTURE}

In our research, we have presented a method for automatic pass thought authentication system based on EEG signals analysis of the brain mental tasks. The proposed method was tested on EGG signals from BCI Competition III dataset. We carried out experiments using 12 signals with different mental tasks. To extract texture features, DWT were used to capture the information of the mental tasks. ANN algorithm was used to classify features into unique classes.

Extensive experiments demonstrate that our method has achieved a good degree of accuracy: for DWT feature extraction algorithm with ANN classifier 98\%, When considering the worst mental tasks features extraction algorithm accuracy dropped to $63 \%$ in ANN classifier. From our experiment we observed that mostly the beta and the theta give better results compared to the alpha. This prompts us to make the hypothesis that the beta and the theta could be further investigated for the characterization of transforming the pass thought classification. In future works we would like to extend this work by adding more features like variance, skewness and kurtosis features to further improve the accuracy of pass thought classification. It will also be interesting to increase the number of classes to cover the commonly accepted major mental tasks groups on human brain signals. Also, a exploring an additional categories of tasks to examine the robustness of brainwave-based authentication against impersonation attacks.

\section{REFERENCES}

[1] Sallam, Amer A., Muhammad Nomani Kabir, Abdulghani Ali Ahmed, Khalid Farhan, and Ethar Tarek. "Epilepsy Detection from EEG Signals Using Artificial Neural Network." In International Conference on Intelligent Computing \& Optimization, pp. 320-327. Springer, Cham, 2018.

[2] Saulynas, Sidas, Charles Lechner, and Ravi Kuber. "Towards the use of brain-computer interface and gestural technologies as a potential alternative to PIN authentication." International Journal of HumanComputer Interaction 34, no. 5 (2018): 433-444.

[3] Zheng, Qingqing, Fengyuan Zhu, and Pheng-Ann Heng. "Robust Support Matrix Machine for Single Trial EEG Classification." IEEE Transactions on Neural Systems and Rehabilitation Engineering 26, no. 3 (2018): 551-562.

[4] Tanyildizi, Harun. "Prediction of the Strength Properties of Carbon Fiber-Reinforced Lightweight Concrete Exposed to the High Temperature Using Artificial Neural Network and Support Vector Machine." Advances in Civil Engineering 2018 (2018).

[5] Zhang, Yong, Bo Liu, Xiaomin Ji, and Dan Huang. "Classification of EEG signals based on autoregressive model and wavelet packet decomposition." Neural Processing Letters 45, no. 2 (2017): 365-378.

[6] Shoeb, Ali Hossam. "Application of machine learning to epileptic seizure onset detection and treatment." PhD diss., Massachusetts Institute of Technology, 2009.

[7] Moran, Lauren V., and L. Elliot Hong. "High vs low frequency neural oscillations in schizophrenia." Schizophrenia bulletin37, no. 4 (2011): 659-663.

[8] Johnson, Benjamin, Thomas Maillart, and John Chuang. "My thoughts are not your thoughts." In Proceedings of the 2014 ACM International Joint Conference on Pervasive and Ubiquitous Computing: Adjunct Publication, pp. 1329-1338. ACM, 2014.

[9] Chuang, John, Hamilton Nguyen, Charles Wang, and Benjamin Johnson. "I think, therefore i am: Usability and security of authentication using brainwaves." In International Conference on Financial Cryptography and Data Security, pp. 1-16. Springer, Berlin, Heidelberg, 2013.

[10] Baby, M. Sneha, A. J. Saji, and C. Sathish Kumar. "Parkinsons disease classification using wavelet 
transform based feature extraction of gait data." In Circuit, Power and Computing Technologies (ICCPCT), 2017 International Conference on, pp. 16. IEEE, 2017.

[11] Kumar, Pradeep, Rajkumar Saini, Partha Pratim Roy, and Debi Prosad Dogra. "A bio-signal based framework to secure mobile devices." Journal of Network and Computer Applications 89 (2017): 62 71.

[12] Curran, Max T., Jong-kai Yang, Nick Merrill, and John Chuang. "Passthoughts authentication with low cost EarEEG." In Engineering in Medicine and Biology Society (EMBC), 2016 IEEE 38th Annual International Conference of the, pp. 1979-1982. IEEE, 2016.

[13] Hosni, Sarah M., Mahmoud E. Gadallah, Sayed F. Bahgat, and Mohamed S. AbdelWahab. "Classification of EEG signals using different feature extraction techniques for mental-task BCI." In Computer Engineering \& Systems, 2007. ICCES'07. International Conference on, pp. 220-226. IEEE, 2007.

[14] Ernawan, F. and Kabir, M.N.: A Robust Image Watermarking Technique with an Optimal DCTPsychovisual Threshold. IEEE Access. 6, 20464-20480 (2018).

[15] Ernawan, F. and Kabir, M.N.: A Blind Watermarking Technique using Redundant Wavelet Transform for Copyright Protection. In: 14th International Colloquium on Signal Processing \& Its Applications (CSPA), pp. 221--226. IEEE (2018).

[16] http://www.bbci.de/competition/iii/ available on 29/12/2018. 